\title{
Social relationship and hair cortisol level in captive male chimpanzees (Pan troglodytes)
}

\section{$\operatorname{AUTHOR}(\mathrm{S}):$}

Yamanashi, Yumi; Teramoto, Migaku; Morimura, Naruki; Nogami, Etsuko; Hirata, Satoshi

\section{CITATION:}

Yamanashi, Yumi .... et al]. Social relationship and hair cortisol level in captive male chimpanzees (Pan troglodytes). Primates 2018, 59(2): 145152

\section{ISSUE DATE:}

2018-3

URL:

http://hdl.handle.net/2433/235529

\section{RIGHT:}

This is a post-peer-review, pre-copyedit version of an article published in 'Primates'. The final authenticated version is available online at: https://doi.org/10.1007/s10329-017-0641-8; The full-text file will be made open to the public on 15 December 2018 in accordance with publisher's 'Terms and Conditions for Self-Archiving'; この論文は出版社版でありま せん。引用の際には出版社版をご確認ざ利用ください。; This is not the published version. Please cite only the published version. 
1 Social relationship and hair cortisol level in captive male chimpanzees (Pan troglodytes)

2

3 Yumi Yamanashi ${ }^{12}$, Migaku Teramoto ${ }^{2}$, Naruki Morimura ${ }^{2}$, Etsuko Nogami ${ }^{2}$, Satoshi

4 Hirata $^{2}$

5 1. Kyoto City Zoo

6 Postal address: Okazaki kouen, Okazaki hosshoujicho, Sakyo-ku, Kyoto City, Japan, 606-

$7 \quad 8333$

8 2. Wildlife Research Center, Kyoto University

9 Postal address: 2-24, Tanaka-sekiden-cho, Sakyo-ku, Kyoto City, Japan, 606-8203

10

$11 *$ Corresponding author

12 Yumi Yamanashi

13 Postal address: Okazaki kouen, Okazaki hosshoujicho, Sakyo-ku, Kyoto City, Japan, 606-

$14 \quad 8333$

15 Email: yumi.yamanashi.kycz@gmail.com

16 Phone: +81-75-771-0211

17 Fax: +81-75-752-1974

18 ORCID digit: 
19

\section{Abstract}

Understanding how social relationships affect long-term stress is important because stress has a profound impact on the welfare of animals and social relationships often exert a strong influence on their stress responses. The purpose of this study was to investigate the relationship between social behaviors and long-term stress levels as assessed by hair cortisol (HC) concentration. The subjects were 11 chimpanzees living in an all-male group (divided into two sub-groups) in Kumamoto Sanctuary, Kyoto University, Japan. Behavioral data were collected between December 2014 and March 2015. The total observation time was 129 h. Hair samples were collected in late March and early April 2015, and cortisol was extracted from the hair and measured with enzyme immunoassay. The hair growth rate was estimated to be $1.33 \pm 0.06 \mathrm{~cm} / \mathrm{month}$. The results revealed that there was a positive correlation between the rate of receiving aggression and HC levels. We also found a significant negative correlation between the balance between giving and receiving grooming (grooming balance index: GBI), which was calculated by subtracting the rate with which grooming is given from that with which it is received, and the rate of receiving aggression and between the GBI and HC levels. Thus, individuals receiving higher levels of aggression also tended to give grooming for relatively long periods compared to the time they were being groomed. In contrast, the rate of initiating aggression did not have a relationship with either HC levels or any measure of social grooming. We did not find social buffering effects, as there was no correlation between mutual social grooming and HC levels. These results show that not only aggressive interactions but also overall social situations in which animals do not have balanced relationships with others might result in the long-term elevation of cortisol levels in captive male chimpanzees. 
Yamanashi 3

\section{$44 \quad$ Keywords}

45 Social behavior; Hair cortisol; Chimpanzee; Long-term stress; Animal Welfare 46 


\section{Introduction}

Social relationships can affect the physical and psychological health of animals including rodents, non-human primates and humans (For review: Cohen 2004; Hennessy et al. 2009). Many studies have suggested that social status and social bonds can affect health, longevity, and stress responses of animals such as baboons, marmoset, squirrel monkeys, rhesus monkeys and chimpanzees (Abbott et al. 2003; Sapolsky 2005; Silk et al. 2010; Wittig et al. 2016). Understanding how social relationships affect long-term stress is important, as long-term stress profoundly affects the health and behavior of animals (Broom and Johnson 1993) and social relationships often exert a strong influence on their stress responses. However, the link between long-term stress and social relationships is not well understood in primates, including our closest relative the chimpanzee, partly due to the difficulty in estimating long-term stress efficiently.

Hair cortisol (HC) has been shown to be a useful measure of long-term hypothalamic-pituitary-adrenal (HPA) axis activation in several species recently (Davenport et al. 2006; Mastromonaco et al. 2014; Russell et al. 2012; Terwissen et al. 2013). The relationship between social relationships and HC in non-human primates has recently been investigated using the novel technique for estimating long-term stress (Tennenhouse et al. 2017; Wooddell et al. 2017). Our studies were the first to apply this methodology to explore this relationship in captive chimpanzees, and suggested that there is a link between aggressive behavior and HC levels in this primate (Yamanashi et al. 2013; Yamanashi et al. 2016a). We found that the rate of receiving aggression positively correlated with HC levels, while that of initiating aggression negatively correlated with HC levels in male chimpanzees. Although a previous study reported that single aggressive interactions in both directions (initiating or receiving) can increase urinary glucocorticoid levels (Wittig et al. 2015), our study showed different effects of 
initiating and receiving aggressive interactions on HC levels in captive chimpanzees (Yamanashi et al. 2016a). Compared with urinary glucocorticoid levels (Bahr et al. 2000), HC levels can reflect long-term accumulation of cortisol over several months (Davenport et al. 2006; Russell et al. 2012). As individuals undergo various experiences daily in addition to aggression, one possible explanation for the different results is that not only aggressive interactions but also other aspects of social life, such as how individuals form affiliative relationships with others, can simultaneously affect longterm accumulation of stress. A previous study reported the stress-reducing effects of grooming with bonding partners (Wittig et al. 2016). Another study suggested that factors such as availability of social supports possibly influence the relationship between social status and measures of stress physiology (Abbott et al. 2003). Our previous study (Yamanashi et al. 2016a) included only aggressive interactions as a measure of social relationships and did not take relationships assessed by affiliative social behaviors into account. Thus, it is unknown whether other social behavior is also related to HC levels, and detailed mechanisms underlying the association between social relationship and long-term stress levels remain unknown. The quality of social relationships might differ between individuals receiving higher levels of aggression and those who often initiate aggressive interactions, when it is assessed by affiliative social behaviors such as social grooming and social play.

Understanding the above-mentioned points is important to improve the quality of care for captive chimpanzees. Wild chimpanzees form a multi-male and multifemale society, which is characterized as a fission-fusion society, and male chimpanzees form strong social bonds (Goodall 1986). Therefore, in animals kept in a captive environment, social groups that are comparable to their wild counterparts should be created, providing chimpanzees an opportunity to develop and exert their 
social skills (AZA Ape Taxon Advisory Group 2010; Ross et al. 2009). Nevertheless, because males are aggressive in nature, keeping several adult males in a captive environment with females is difficult as it often results in escalated aggression. As a result, the formation of social groups including only one or two males and multiple females is more frequent. Unfortunately, because of this practice of group formation, surplus males often emerge and in the worst case, these surplus individuals can become socially isolated. Providing a social environment for all captive chimpanzees and methodologies by which this can be achieved are among the most important topics related to the welfare of chimpanzees. One approach to providing a social environment to surplus male chimpanzees is the formation of all-male groups, although in wild chimpanzees, such groups do not exist, unlike the case for wild gorillas, which sometimes form groups of this kind (Fritz and Howell 2001; Yamanashi et al. 2016a). However, the types of social relationships that males in all-male groups form and how this affects long-term stress responses in captive environments with limited opportunities for fission are not well understood. Investigating such points is useful for creating guidelines for the formation and management of social groups including multiple male chimpanzees and for discussing optimal strategies for the organization of social groups for captive chimpanzees.

The purpose of this study was to investigate the relationship between social behavior and long-term stress levels assessed by HC concentration. We tested the following three hypotheses. Our hypothesis is that individuals who receive higher levels of aggression need to invest more of their time in forming stable relationships and avoiding future aggression on a daily basis, which results in an increase in stress over a long period of time (the investment hypothesis). In contrast, individuals who often initiate aggression would not need to engage in such activities. We predicted that there 
122 would be an association between aggression and giving grooming and thus also an association between HC levels and the rate of giving grooming. We also hypothesized that individuals who had good relationships with others would show lower levels of HC based on social buffering theory (Kikusui et al. 2006). We predicted that the rate of mutual grooming could have a negative relationship with $\mathrm{HC}$ levels and that the rate of social play would not show any relationship with HC levels. This is because our previous study (Yamanashi et al. 2017) showed that the rate of mutual grooming implied an affiliative relationship with others, but social play did not imply such a relationship in the same population of chimpanzees. Finally, we hypothesized that a balance between giving and receiving grooming is also important (grooming balance hypothesis). Costs accrue when individuals groom others, while they gain benefits from receiving grooming (Machanda et al. 2014). If individuals invest time in giving grooming, but also receive grooming, costs might be balanced to some extent by the benefits gained from receiving grooming. Therefore, rather than only considering the rate with which social grooming is given, the balance between giving and receiving grooming can be more important. We used the grooming balance index (GBI) to test this hypothesis. The GBI is simply the subtraction of the rate with which grooming is given from that with which it is received. This value increases if an individual receives more

140 unilateral social grooming than what is given. When assessing the extent of dyad-level grooming equality, previous studies have often utilized the grooming equality index

142 (GEI), which also includes differences in the length of time grooming is given and received but that is adjusted by the total time of grooming given and received (e.g. Mitani 2009; Silk et al. 2013). This index might also be useful for checking the grooming balance at the individual level, but a problem arises when considering the

146 situation in captive chimpanzees. It is well known that like other primates, captive 
chimpanzees often show social impairment due to the environment in which their early rearing took place. The level of engagement in social grooming decreases if they had less experience with conspecifics as infants (Freeman and Ross 2014). The GEI is between 0 and 1 , and information on the absolute amount of time individuals invest in grooming is eliminated. As a result, the value can be similar between individuals who are social and invest much time in giving grooming but who do not receive grooming and those who are less social and devote less time to giving grooming and who do not receive grooming. Therefore, we used the GBI, which is similar but differs in the value not being adjusted by the total time of grooming given and received. We collected equal amounts of data from each individual, so possible biases arising from inter-individual differences in observation time could be minimized. We used this simple, but new, index to test the final hypothesis. We predicted that individuals who receive high levels of aggression also receive less grooming while they give more grooming.

\section{Methods}

The subjects were 11 chimpanzees living in an all-male group in Kumamoto Sanctuary, Kyoto University, Japan (KS). Males in the all-male groups of chimpanzees were biologically unrelated except for one dyad that had an uncle-nephew relationship. Ages of the individuals were written in (Supplementary1). KS is the sanctuary for chimpanzees and bonobos in Japan (for details of the sanctuary, please see Morimura et al. (2010)). All individuals had access to 14 indoor and six outdoor cages. Each indoor cage was $7 \mathrm{~m}^{2}$ in area and $2.6 \mathrm{~m}$ in height. The six outdoor cages included four small cages (approximately 23-34 $\mathrm{m}^{2}$ in area and approximately $4 \mathrm{~m}$ in height) and two large cages (120 $\mathrm{m}^{2}$ in area and $12 \mathrm{~m}$ in height; $110 \mathrm{~m}^{2}$ in area and $8 \mathrm{~m}$ in height). All these cages were connected with each other and could be connected or disconnected at any 
time. Based on these flexible joint cages, the 11 chimpanzees were divided into two subgroups during the observation. The members of each subgroup were periodically changed to provide social stimulation and prevent escalated aggression, particularly directed toward immigrant individuals (Yamanashi et al. 2016a). Therefore, the number of chimpanzees in each subgroup was between 4 and 7, depending on the day during this study period. The chimpanzees had free access to water at any time, and regular meals (consisting mainly of fruits, vegetables, and monkey pellets) were provided three times per day. Additionally, routine feeding enrichment (e.g., juice feeders, puzzle feeders, browsing opportunities, and foods concealed in boxes or newspapers) were changed daily. Other types of environmental enrichment were also provided. For example, fire hoses, ropes, hammocks, climbing structures, and substrate materials were installed, and natural vegetation was planted to increase the complexity of the physical environment. Spaces were also available for the chimpanzees to escape from rain, strong sunlight, and cold, and they were provided with comfortable bedding materials for day- and night-time sleep. Materials that they could manipulate freely were also provided, such as toys, buoys, and sacks.

This study was approved by the ethics committee of the Wildlife Research Center, Kyoto University (No. WRC-2014KS001A). We have complied with the ethical standards for the treatment of the chimpanzees according to the guidelines of the Primate Society of Japan, which adhered to the legal requirement of Japan.

\section{Data collection}

Behavioral data were collected between December 2014 and March 2015. The total observation time was $129 \mathrm{~h}$. All data were collected by YY, using the iOS application “ISBOapp” and a notebook to complement (Ogura 2013). Between 11:00 
and 15:00, YY conducted 30 min of focal observations of 11 chimpanzees in a randomly assigned order and recorded behaviors of the chimpanzees in their enclosures (Martin and Bateson 2007). At least 10.5 h (21-23 sessions of focal observation) of focal animal sampling were conducted for each individual, and the observation sessions for each individual were almost evenly distributed between 11:00 and 15:00. During the focal observations, YY recorded social grooming (Fig 1), pant-grunt and social play every 30 s and all aggressive interactions that occurred within the groups (Table 1). YY stopped making focal observations and began recording aggressive interactions when a scream was heard or when there were any other signs of aggression. YY recorded the aggressors and receivers of each aggressive interaction.

\section{Hair cortisol analysis}

The methods to quantify HC levels were based on our previous study (Yamanashi et al. 2016a; Yamanashi et al. 2016b). In these studies, we validated HC analysis methods and investigated various analysis methods to obtain stable results. To collect hair samples, EN cut the arm hairs of the 11 chimpanzees with scissors. Although the hair was attempted to cut at the skin surface as much as possible, few mm of hair often remained. Moreover, small variations existed in terms of the location of hair sample collection across the chimpanzees. Our previous study indicated that we can obtain consistent results if hair samples are collected from similar body parts (Yamanashi et al. 2016b). Hair samples were collected in late March and early April 2015 after completing the behavioral data collection. We cut the arm hair once and waited the regrowth of hair to evaluate the hair growth rate in five captive male chimpanzees in the KS. We cut the regrown hairs twice for each individual after approximately one and two months from the first cut. We randomly selected 10 hairs 
from each sample and measured the length. The growth rate was adjusted by the number of days following the haircut and we took the individual average from data obtained after one and two months. The average hair growth rate was $1.33 \pm 0.06 \mathrm{~cm} / \mathrm{month}$ (mean $\pm \mathrm{SD}$ ). Our previous study showed that the average hair length was $5.86 \pm$ $1.83 \mathrm{~cm}$ (mean $\pm \mathrm{SD}$ ) and no effect of degradation along the hair shaft (Yamanashi et al. 2013). Therefore, we determined that cortisol was accumulated for previous 4-5 months in the full hair length and that the period of behavioral data collection roughly corresponded to the hair sampling period. Due to the slight individual variations in the hair growth rate and hair length, we used full hair length for analysis rather than using identical hair lengths across the animals. Additional details of the $\mathrm{HC}$ analysis methods are described in our previous study (Yamanashi et al. 2016b). Cortisol concentrations were measured with a salivary cortisol enzyme immunoassay (EIA) kit (Salimetrics, Philadelphia, PA, USA). The sensitivity of the kit was $<0.03 \mathrm{ng} / \mathrm{ml}$, and the coefficient of variation within a plate was $4.08 \%$.

\section{Data analysis}

We used the average rate of each social behavior to investigate the relationship with HC levels. We also used the difference between grooming given and received as a measure of the level of investment toward others (Grooming Balance Index, GBI: rate of grooming received minus rate of grooming given). Although we tried to quantify the ranks of the individuals by the direction of pant grunting (Nishida et al. 2010; Noe et al. 1980), we could identify only the two highest- and two lowest-ranking individuals. The group members directed pant grunts toward the alpha male, and the lowest-ranking individuals directed pant grunts toward several individuals. However, there were many blank relationships and other individuals did not pant-grunt at each other. Thus, we were 
unable to quantify the rank of every individual. Nevertheless, aggression was seldom directed at highest-ranking individuals and frequently directed at lowest-ranking individuals (Supplementary 1, 2). Therefore, the rate of being subjected to aggression was closely related to the dominance rank of these chimpanzees.

\section{Statistical analysis}

Relationships between aggressive behaviors and affiliative behaviors and between each social behavior and HC levels were tested by Spearman's rank correlation test. All statistical testing was conducted with R. 3.3.3 (R Development Core Team 2017). We used the package “coin” to calculate $p$-values for Spearman’s rank correlation test (Hothorn et al. 2008). The level of significance was set at $\alpha=0.05$.

\section{Results}

\section{Association between aggression and other social behaviors}

There was a significant negative correlation between the rate of receiving aggression and GBI ( $\rho=-0.65, Z=-2.04, p=0.0412)$. The rates of initiating aggression, mutual grooming, giving and receiving unilateral grooming, and social play were not significantly correlated with the rate of receiving aggression (initiating aggression: $\rho=0.187, Z=0.591, p=0.555$; giving unilateral grooming: $\rho=0.436, Z=$ 1.38, $p=0.168$; receiving unilateral grooming: $\rho=-0.227, Z=-0.719, p=0.472$; mutual grooming: $\rho=-0.182, Z=-0.575, p=0.565$; social play: $\rho=0.178, Z=0.562$, $p=0.574)$.

Correlation between the rate of initiating aggression and social play was marginally significant ( $\rho=0.605, Z=1.91, p=0.0557$ ). Other behaviors (the rates of mutual grooming, giving and receiving unilateral grooming, and GBI) did not show any 
correlation with the rate of initiating aggression (mutual grooming: $\rho=-0.356, Z=-$ 1.12, $p=0.261$; giving unilateral grooming: $\rho=-0.0456, Z=-0.144, p=0.885$; receiving unilateral grooming: $\rho=-0.105, Z=-0.331, p=0.740$; GBI: $\rho=-0.0091, Z=$ $-0.0288, p=0.977)$.

\section{Association between social behaviors and hair cortisol}

We found that the rate of receiving aggression was positively correlated with HC levels (Fig 2-a: $\rho=0.71, Z=2.23, p=0.0255$ ). We also found a significant negative correlation between HC levels and GBI (Fig 2-c: $\rho=-0.78, Z=-2.38, p=0.0175$ ).

Correlation between HC levels and the rate of giving unilateral grooming was marginally significant ( $\rho=0.59, Z=1.86, p=0.0631$ ). The rates of initiating aggression, receiving unilateral social grooming, mutual grooming, and social play were not significantly correlated (Fig 2-b: initiating aggression: $\rho=-0.22, Z=-0.708, p=$ 0.479; receiving unilateral grooming: $\rho=-0.18, Z=-0.56, p=0.574$; Fig 2-d: mutual grooming: $\rho=0.28, Z=0.89, p=0.372$; Fig 2-e: social play: $\rho=-0.03, Z=-0.10, p=$ $0.920)$. There was no significant correlation between age and HC levels ( $\rho=0.04, Z=$ $0.13, p=0.896)$.

\section{Discussion}

The results of our study supported our prediction for the grooming balance hypothesis. We found a positive correlation between the rate of receiving aggression and HC levels, as in the previous studies in male chimpanzees (Yamanashi et al. 2013; Yamanashi et al. 2016a). In this study, we also investigated the relationship between affiliative behaviors and $\mathrm{HC}$ levels for the first time. We found a significant negative correlation between GBI and the rate of receiving aggression, and between GBI and HC 
levels. In contrast, the rate of initiating aggression did not have a relationship with either HC levels or social grooming. Thus, individuals receiving higher levels of aggression also showed skewed patterns of social grooming, in which they have to invest their time in giving grooming while they do not receive grooming from others so often. Situations like these might cause long-term elevations in cortisol levels in such individuals. Although we investigated the relationship between the rate of giving grooming and $\mathrm{HC}$ levels to test the investment hypothesis, we found the trend to be only marginally significant. Therefore, although it is still possible that it is costly to spend much time on giving grooming to maintain relationships with others, the balance is more important. As there was a significant correlation between the rate of receiving aggression and the GBI, overall social situations in which chimpanzees often receive aggression and have to pay a higher cost for maintaining social relationships might result in increased stress levels in such individuals in the long term.

A study in a wild population of chimpanzees showed a positive correlation between dominance and fecal glucocorticoid metabolites (Muller and Wrangham 2004). The finding of our present study in terms of the correlation between aggression and cortisol is opposite, considering the fact that the study of Muller and Wrangham assessed dominance relationships based on aggressive interactions, and the close relationship between dominance rank and the rate of being subjected to aggression in our study chimpanzees. Studies have suggested that the relationship between dominance and stress can differ even in the same species; the possible explanations offered were social stability, physical environment, and controllability of the surrounding environment (Abbott et al. 2003). During the period of the present study, there were no obvious hierarchical changes in the studied group. The results suggested that there was no need for dominant individuals to make efforts to maintain their rank, while 
individuals who received high levels of aggression needed to work hard on a daily basis. The nature of the social relationship that dominant individuals form with others can vary depending on the situation and group characteristics. Therefore, to understand the effects of social environment on long-term stress responses, it is necessary to investigate multiple social behaviors and quantify detailed situations of individuals.

Our prediction for the social buffering hypothesis was not supported. We did not find any relationship between the rate of mutual social grooming and HC levels. We also did not find any significant relationship between the rate with which grooming was received and HC levels. Social play also did not show a significant correlation with HC levels. Although there is still a possibility that mutual social grooming or receiving grooming buffer stress for short periods, as suggested by a previous study (Wittig et al. 2016), the results of this study indicated that receiving aggression and grooming balance had a stronger effect when the accumulation of stress over long periods was considered. Some aspects of this study limit understanding the relationship between social situations and long-term stress levels. For example, we used the GBI to analyze the association between hair cortisol level and grooming balance. The GBI only considers differences in the overall rates with which grooming is given and received. Therefore, we could not explain how the social relationship with every other individual contributed to the stress responses of these chimpanzees. It might be sufficient to have a strong social bond with one partner from whom the individual can receive grooming, but having multiple moderate affiliative bonds might be better for having a less stressful life. The small sample size in this study did not allow us to investigate this point as the situation of each chimpanzee varied a lot. In the future, social relationships and stress levels should be investigated in multiple captive social groups. 
chimpanzees living in an all-male group. Not only aggressive interactions but also social situations in which chimpanzees do not have balanced relationships with others with whom they interact on a daily basis might result in long-term elevations in cortisol levels in captive male chimpanzees. Chimpanzees which had to invest their time in giving grooming without being groomed showed higher levels of stress. Aggression and incompatible combinations of individuals are often inevitable in captive chimpanzee groups, particularly when the group size is large. Therefore, group formation should be decided on taking the situation of each chimpanzee into consideration to ensure that each chimpanzee can have balanced relationships with others. In our sanctuary, we were aware of this and rotated the members of the all-male group to prevent escalated aggression, particularly directed toward immigrant individuals and excessive stress on such individuals. They are provided with opportunities to occasionally split up and maintain an appropriate distance from each other. Nevertheless, social situations had a strong influence on the stress level in male chimpanzees. Future studies should investigate solutions to alleviate the stress of such individuals and monitor such high HC individuals to check the effects later in life.

\section{Acknowledgments}

The care of the chimpanzees and the present study were supported financially by grants from the Japan Society for the Promotion of Science (\#13J04636 and \#17K17828 to YY, \#25119008 to SH, \#15H0579, \#16H06301, \#16H06283, JSPS-LGPU04, JSPS core-to-core CCSN) and also by Future Development Funding Program of Kyoto University Research Coordination Alliance and Great Ape Information Network. We are grateful to the following people and program for their support of our study: Yusuke Mori, Toshifumi Udono and the staff at the Kumamoto Sanctuary, and Great 
372 Ape Information Network. 


\section{References}

Abbott DH et al. (2003) Are subordinates always stressed? A comparative analysis of rank differences in cortisol levels among primates Horm Behav 43:67-82 doi:10.1016/s0018-506x(02)00037-5

Bahr NI, Palme R, Mohle U, Hodges JK, Heistermann M (2000) Comparative aspects of the metabolism and excretion of cortisol in three individual nonhuman primates Gen Comp Endocrinol 117:427-438

Broom DM, Johnson KG (1993) Stress and animal welfare. Chapman \& Hall Animal Behaviour Series. Chapman \& Hall, London

Cohen S (2004) Social relationships and health Am Psychol 59:676

Davenport MD, Tiefenbacher S, Lutz CK, Novak MA, Meyer JS (2006) Analysis of endogenous cortisol concentrations in the hair of rhesus macaques Gen Comp Endocrinol 147:255-261

Freeman HD, Ross SR (2014) The impact of atypical early histories on pet or performer chimpanzees PeerJ 2:e579 doi:10.7717/peerj.579

Fritz J, Howell S (2001) Captive chimpanzee social group formation. In: Brent L (ed) The care and management of captive chimpanzees. vol 2. American Society of Primatologists, San Antonio, pp 173-204

Goodall J (1986) The chimpanzees of Gombe: patterns of behaviour. Harvard University Press, Cambridge

Hennessy MB, Kaiser S, Sachser N (2009) Social buffering of the stress response: diversity, mechanisms, and functions Front Neuroendocrinol 30:470-482 doi:10.1016/j.yfrne.2009.06.001

Hothorn T, Hornik K, van de Wiel MA, Zeileis A (2008) Implementing a class of permutation tests: the coin package Journal of Statistical Software 28:1-23

Kikusui T, Winslow JT, Mori Y (2006) Social buffering: relief from stress and anxiety Philos Trans R Soc Lond B Biol Sci 361:2215-2228

Machanda ZP, Gilby IC, Wrangham RW (2014) Mutual grooming among adult male chimpanzees: the immediate investment hypothesis Anim Behav 87:165-174 doi:http://dx.doi.org/10.1016/j.anbehav.2013.10.028

Martin P, Bateson P (2007) Measuring behaviours: an introductory guide 3rd edn. Cambridge University Press, Cambridge

Mastromonaco GF, Gunn K, McCurdy-Adams H, Edwards DB, Schulte-Hostedde AI (2014) Validation and use of hair cortisol as a measure of chronic stress in eastern chipmunks (Tamias striatus) Conservation Physiology 2 doi:10.1093/conphys/cou055 
409

410

411

412

413

414

415

416

417

418

419

420

421

422

423

424

425

426

427

428

429

430

431

432

433

434

435

436

437

438

439

440

441

442

443

444

445

Mitani JC (2009) Male chimpanzees form enduring and equitable social bonds Anim Behav 77:633-640 doi:http://dx.doi.org/10.1016/j.anbehav.2008.11.021

Morimura N, Idani G, Matsuzawa T (2010) The first chimpanzee sanctuary in Japan: an attempt to care for the "surplus" of biomedical research Am J Primatol 73:226-232 doi:10.1002/ajp.20887

Muller MN, Wrangham RW (2004) Dominance, cortisol and stress in wild chimpanzees (Pan troglodytes schweinfurthii) Behavioral Ecology and Sociobiology 55:332-340 doi:10.1007/s00265-003-0713-1

Nishida T, Zamma K, Matsusaka T, Inaba A, McGrew WC (2010) Chimpanzee behavior in the wild: an audio-visual encyclopedia. Springer-Verlag, Tokyo

Noe R, de Waal FB, Van Hooff J (1980) Types of dominance in a chimpanzee colony Folia Primatol (Basel) 34:90-110

Ogura T (2013) A digital app for recording enrichment data The Shape of Enrichment 22:3

R: A language and environment for statistical computing (2017) http://www.R-project.org/.

Russell E, Koren G, Rieder M, Van Uum S (2012) Hair cortisol as a biological marker of chronic stress: Current status, future directions and unanswered questions Psychoneuroendocrinology 37:589-601

Sapolsky RM (2005) The influence of social hierarchy on primate health Science 308:648652 doi:10.1126/science. 1106477

Silk J, Cheney D, Seyfarth R (2013) A practical guide to the study of social relationships Evolutionary Anthropology: Issues, News, and Reviews 22:213-225 doi:10.1002/evan.21367

Silk JB et al. (2010) Strong and consistent social bonds enhance the longevity of female baboons Curr Biol 20:1359-1361 doi:http://dx.doi.org/10.1016/j.cub.2010.05.067

Tennenhouse EM, Putman S, Boisseau NP, Brown JL (2017) Relationships between steroid hormones in hair and social behaviour in ring-tailed lemurs (Lemur catta) Primates 58:199-209 doi:10.1007/s10329-016-0566-7

Terwissen CV, Mastromonaco GF, Murray DL (2013) Influence of adrenocorticotrophin hormone challenge and external factors (age, sex, and body region) on hair cortisol concentration in Canada lynx (Lynx canadensis) Gen Comp Endocrinol 194:162-167 doi:http://dx.doi.org/10.1016/j.ygcen.2013.09.010

Wittig RM, Crockford C, Weltring A, Deschner T, Zuberbühler K (2015) Single aggressive interactions increase urinary glucocorticoid levels in wild male chimpanzees PLoS One 10:e0118695 doi:10.1371/journal.pone.0118695

Wittig RM, Crockford C, Weltring A, Langergraber KE, Deschner T, Zuberbühler K (2016) Social support reduces stress hormone levels in wild chimpanzees across stressful events and everyday affiliations Nature Communications 7:13361 


\section{Yamanashi 20}

446

447

448

449

450

451

452

453

454

455

456

457

458

459

460

461

462

463

464

465

doi:10.1038/ncomms13361

http://www.nature.com/articles/ncomms13361\#supplementary-information

Wooddell LJ et al. (2017) Relationships between affiliative social behavior and hair cortisol concentrations in semi-free ranging rhesus monkeys Psychoneuroendocrinology 84:109-115 doi:https://doi.org/10.1016/j.psyneuen.2017.06.018

Yamanashi Y, Morimura N, Mori Y, Hayashi M, Suzuki J (2013) Cortisol analysis of hair of captive chimpanzees (Pan troglodytes) Gen Comp Endocrinol 194:55-63 doi:http://dx.doi.org/10.1016/j.ygcen.2013.08.013

Yamanashi Y, Nogami E, Teramoto M, Morimura N, Hirata S (2017) Adult-adult social play in captive chimpanzees: is it indicative of positive animal welfare? Applied Animal Behaviour Science doi:https://doi.org/10.1016/j.applanim.2017.10.006

Yamanashi Y, Teramoto M, Morimura N, Hirata S, Inoue-Murayama M, Idani G (2016a) Effects of relocation and individual and environmental factors on the long-term stress levels in captive chimpanzees (Pan troglodytes): monitoring hair cortisol and behaviors PLoS One 11:e0160029 doi:10.1371/journal.pone.0160029

Yamanashi Y et al. (2016b) Analysis of hair cortisol levels in captive chimpanzees: effect of various methods on cortisol stability and variability MethodsX 3:110-117 doi:http://dx.doi.org/10.1016/j.mex.2016.01.004 
466 Figure Caption

467 Fig 1. Photograph of social grooming among three males.

468 Mutual social grooming (a); Unilateral social grooming (b)

469

470 Fig 2. Relationship between social behaviors and HC concentration (pg/mg hair)

471 Relationship between the rate of receiving aggression and HC levels (a); the rate of

472 initiating aggression and HC levels (b); GBI (difference between grooming received and

473 given; Gr received minus Gr given) and HC levels (c); the rate of mutual social

474 grooming and HC levels (d); and the rate of social play and HC levels (e).

475

476

477 


\section{Fig1}

(a)

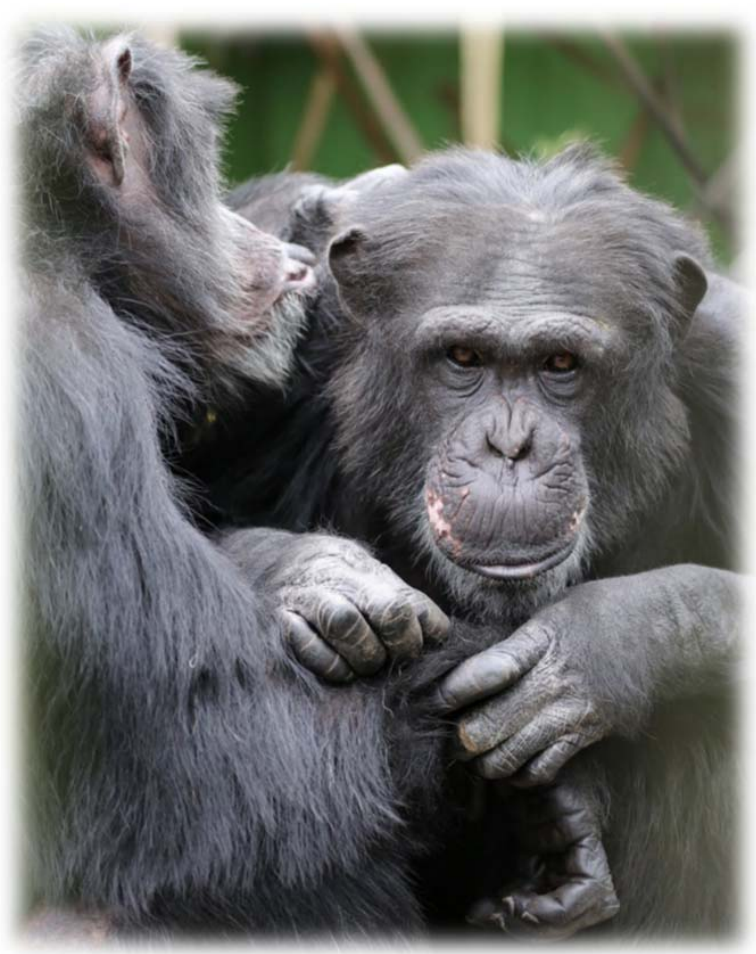

(b)

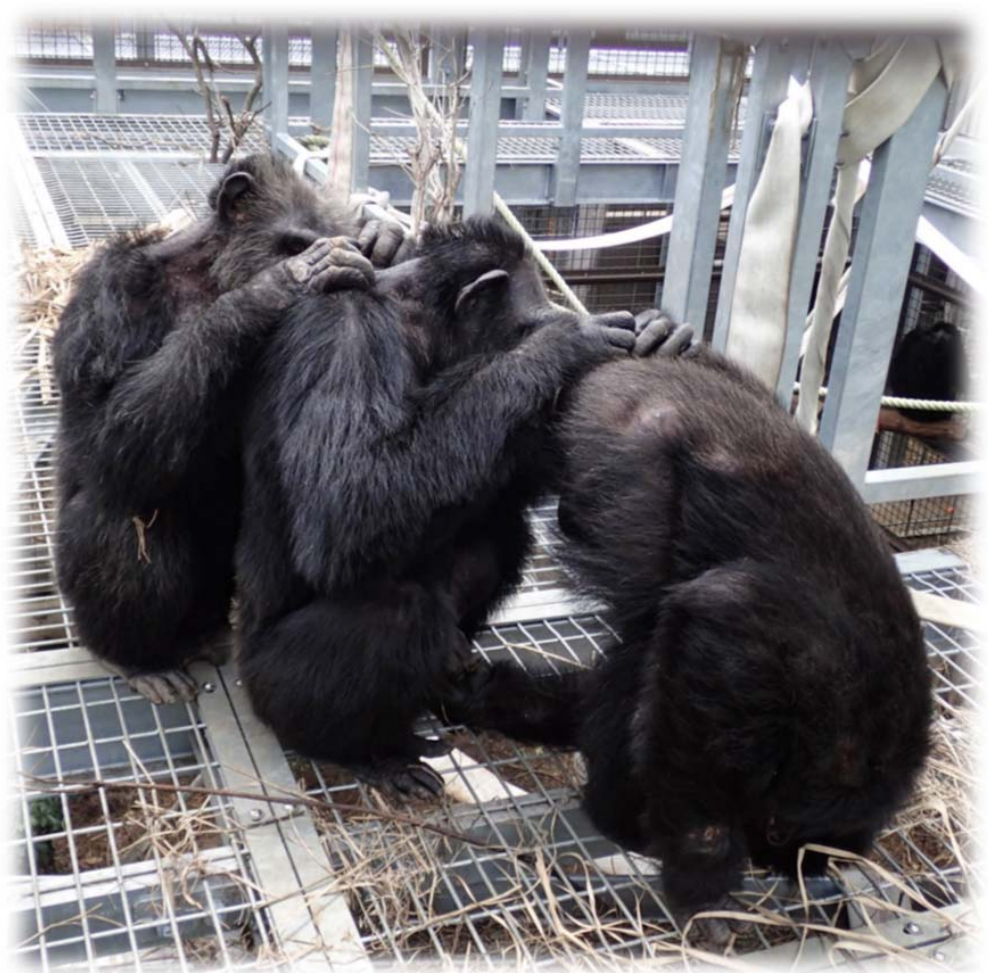



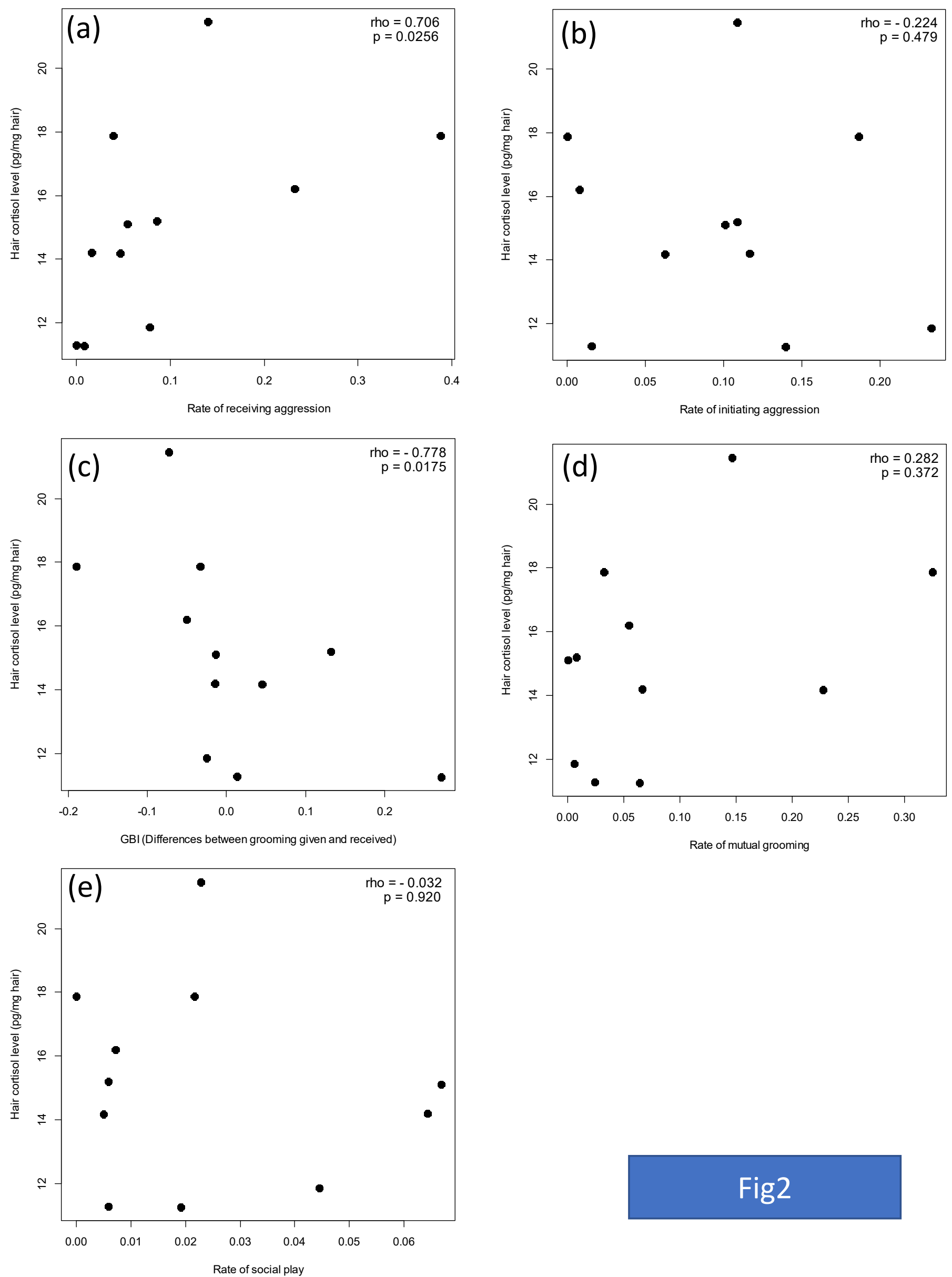

\section{Fig2}


Table 1. Definition of behaviors

\begin{tabular}{ll}
\hline Behaviors & Definitions \\
\hline Bggressive interactions & $\begin{array}{l}\text { Behaviors that included chasing, hitting, biting, kicking, and } \\
\text { charging displays directed toward group members; individuals } \\
\text { who were targets of such behavior showed screaming, } \\
\text { escaping, or counterattacking behaviors. }\end{array}$ \\
& Playing with conspecifics. The behaviors included play push, \\
play bite, play slap, tickle, play run, grab hands or legs & repeatedly and rhythmically, social object play, rough and \\
& tumble and play stamping. These behaviors sometimes \\
& appeared sequentially. Social play is distinguishable from \\
other social behaviours since all the social play sessions in \\
this study included play face or play pants. \\
Social play \\
Groom another with hands or mouth. Mutual grooming \\
included the grooming which two chimpanzees groom each \\
other simultaneously. Groom unilateraly included the \\
grooming another without reciprocation.
\end{tabular}

